\title{
Random Attractors for Stochastic Three-Component Reversible Gray-Scott System on Infinite Lattices
}

\author{
Anhui Gu, ${ }^{1}$ Zhaojuan Wang, $^{2}$ and Shengfan Zhou $^{3}$ \\ ${ }^{1}$ College of Science, Guilin University of Technology, Guilin 541004, China \\ ${ }^{2}$ School of Mathematical Science, Huaiyin Normal University, Huaian 223300, China \\ ${ }^{3}$ Department of Mathematics, Zhejiang Normal University, Jinhua 321004, China
}

Correspondence should be addressed to Anhui Gu, mathgu@yahoo.cn

Received 13 April 2012; Accepted 29 May 2012

Academic Editor: Xiaohui Liu

Copyright (C) 2012 Anhui Gu et al. This is an open access article distributed under the Creative Commons Attribution License, which permits unrestricted use, distribution, and reproduction in any medium, provided the original work is properly cited.

We prove the existence of a compact random attractor for the random dynamical system generated by stochastic three-component reversible Gray-Scott system with a multiplicative white noise on infinite lattices.

\section{Introduction}

Consider the following stochastic three-component reversible Gray-Scott system with a multiplicative white noise on infinite lattices:

$$
\begin{aligned}
& \frac{d u_{i}}{d t}=d_{1}\left(u_{i-1}-2 u_{i}+u_{i+1}\right)-(F+k) u_{i}+u_{i}^{2} v_{i}-G u_{i}^{3}+N w_{i}+u_{i} \circ \frac{d W}{d t}, \\
& \frac{d v_{i}}{d t}=d_{2}\left(v_{i-1}-2 v_{i}+v_{i+1}\right)+F\left(1-v_{i}\right)-u_{i}^{2} v_{i}+G u_{i}^{3}+v_{i} \circ \frac{d W}{d t} \\
& \frac{d w_{i}}{d t}=d_{3}\left(w_{i-1}-2 w_{i}+w_{i+1}\right)+k u_{i}-(F+N) w_{i}+w_{i} \circ \frac{d W}{d t}, \\
& u(0)=u_{0}=\left(u_{i 0}\right)_{i \in \mathbb{Z}}, \quad v(0)=v_{0}=\left(v_{i 0}\right)_{i \in \mathbb{Z},}, \quad w(0)=w_{0}=\left(w_{i 0}\right)_{i \in \mathbb{Z}},
\end{aligned}
$$


where $i \in \mathbb{Z}$ (the set of integers), $\left(u_{i}\right)_{i \in \mathbb{Z}} \in \ell^{2},\left(v_{i}\right)_{i \in \mathbb{Z}} \in \ell^{2}$, and $\left(w_{i}\right)_{i \in \mathbb{Z}} \in \ell^{2}$; all the parameters are positive constants; $W$ is a Brownian motion on $(\Omega, \mathcal{F}, \mathbb{P})$ and $\circ$ denotes the Stratonovich sense of the stochastic term.

System (1.1) can be considered as a discrete model of stochastic three-component reversible Gray-Scott system in which the existence of a random attractor has been established [1]. When there is no stochastic term, system (1.1) can be considered as a discrete analogue of the following three-component reversible Gray-Scott system in $\mathbf{R}$ :

$$
\begin{gathered}
\frac{\partial u}{\partial t}=d_{1} \Delta u-(F+k) u+u^{2} v-G u^{3}+N w, \\
\frac{\partial v}{\partial t}=d_{2} \Delta v+F(1-v)-u^{2} v+G u^{3}, \\
\frac{\partial w}{\partial t}=d_{3} \Delta w+k u-(F+N) w,
\end{gathered}
$$

which was firstly introduced by Mahara et al. [2], then it was reduced to system (1.2) under some nondimensional transformations in You [3]. Also, the existence of a global attractor for the solution semiflow of (1.2) with Neumann boundary condition on a bounded domain of space dimension $n \leq 3$ was proved in [3].

When $G=0$ and $w=0$, system (1.2) becomes the two-components Gray-Scott equations which was one of the models signified the seminal work of the Brussell school. The model originated from describing an isothermal, cubic autocatalytic, continuously fed and diffusive reactions of two chemicals (see [4-8]), but neglected the reversible factors. Indeed, the reversibility in the interactions of multispecies is an indispensable factor in many processes in natural and social sciences. If we take the reversibility into account, it yields system (1.2).

Stochastic lattice differential equations have discrete spatial structures and take random influences into account. These random effects are not only introduced to compensate for the defects in some deterministic models, but are also rather intrinsic phenomena. Bates et al. [9] initiated the consideration of stochastic lattice dynamical systems with additive noises and Caraballo and $\mathrm{Lu} \mathrm{[10]} \mathrm{was} \mathrm{the} \mathrm{first} \mathrm{to} \mathrm{consider} \mathrm{the} \mathrm{stochastic} \mathrm{lattice} \mathrm{dynamical} \mathrm{systems}$ with a multiplicative noise, and Han et al. [11] generalized the results of $[9,10]$ to a more general space. For more details and the quite recent results, we can refer to, for example, [12-15].

Just like the models considered in biology, the discrete time models governed by difference equations are more appropriate than the continuous ones; we can also deal with the chemical and biochemical reactions in the same manner, see, for example, $[16,17]$ and the references therein. However, very few investigations are on this topic, especially for the stochastic three-component reversible Gray-Scott system on infinite lattices, is widely open, to the best of our knowledge.

The paper is organized as follows. In Section 2, we present some preliminaries and definitions. Section 3 is devoted to the existence of a random attractor. 


\section{Preliminaries}

Let $(\Omega, \mathcal{F}, \mathbb{P})$ be a probability space, where $\Omega$ is a subset of $\mathcal{C}_{0}(\mathbf{R}, \mathbf{R})=\{\omega \in \mathcal{C}(\mathbf{R}, \mathbf{R}): \omega(0)=$ $0\}$, which endowed with the compact open topology (see [18]), $\mathcal{F}$ is the Borel $\sigma$-algebra, and $\mathbb{P}$ is the corresponding Wiener measure on $\Omega$. Let $\theta_{t} \omega(\cdot)=\omega(\cdot+t)-\omega(t), t \in \mathbf{R}$, then $\left(\Omega, \mathcal{F}, \mathbb{P},\left(\theta_{t}\right)_{t \in \mathbf{R}}\right)$ is an ergodic metric dynamical system. Throughout the paper, we denote

$$
\ell^{2}=\left\{x=\left(x_{i}\right)_{i \in \mathbb{Z}}: x_{i} \in \mathbf{R}, \sum_{i \in \mathbb{Z}} x_{i}^{2}<\infty\right\}
$$

the Hilbert space equipped with the usual inner product and norm:

$$
\langle x, y\rangle=\sum_{i \in \mathbb{Z}}\left(x_{i}, y_{i}\right), \quad\|x\|^{2}=\langle x, x\rangle, \quad \forall x=\left(x_{i}\right)_{i \in \mathbb{Z},} \quad y=\left(y_{i}\right)_{i \in \mathbb{Z}} \in \ell^{2}
$$

For the reader's convenience, we introduce some basic concepts related to random dynamical systems and random attractor, which are taken from $[11,18,19]$. Let $\left(H,\|\cdot\|_{H}\right)$ be a separable Hilbert space and $\left(\Omega_{0}, \mathcal{F}, \mathbb{P}\right)$ a probability space.

Definition 2.1. A stochastic process $\{\varphi(t, \omega)\}_{t \geq 0, \omega \in \Omega_{0}}$ is a continuous random dynamical system over $\left(\Omega_{0}, \mathcal{F}, \mathbb{P},\left(\theta_{t}\right)_{t \in \mathbf{R}}\right)$ if $\varphi$ is $(\mathbb{B}[0, \infty) \times \mathcal{F} \times \mathbb{B}(H), \mathcal{B}(H))$-measurable, and for all $\omega \in \Omega_{0}$,

(i) the mapping $\varphi(t, \omega): H \mapsto H, x \mapsto \varphi(t, \omega) x$ is continuous for every $t \geq 0$,

(ii) $\varphi(0, \omega)$ is the identity on $H$,

(iii) (cocycle property) $\varphi(s+t, \omega)=\varphi\left(t, \theta_{s} \omega\right) \varphi(s, \omega)$ for all $s, t \geq 0$.

Definition 2.2. (i) A set-valued mapping $\omega \mapsto B(\omega) \subset H$ (we may write it as $B(\omega)$ for short) is said to be a random set if the mapping $\omega \mapsto \operatorname{dist}_{H}(x, B(\omega))$ is measurable for any $x \in H$, where $\operatorname{dist}_{H}(x, D)$ is the distant in $H$ between the element $x$ and the set $D \subset H$.

(ii) A random set $B(\omega)$ is said to be bounded if there exist $x_{0} \in H$ and a random variable $r(\omega)>0$ such that $B(\omega) \subset\left\{x \in H:\left\|x-x_{0}\right\|_{H} \leq r(\omega), x_{0} \in H\right\}$ for all $\omega \in \Omega_{0}$.

(iii) A random set $B(\omega)$ is called a compact random set if $B(\omega)$ is compact for all $\omega \in \Omega_{0}$.

(iv) A random bounded set $B(\omega) \subset H$ is called tempered with respect to $\left(\theta_{t}\right)_{t \in \mathbf{R}}$ if for a.e. $\omega \in \Omega_{0}, \lim _{t \rightarrow+\infty} e^{-\gamma t} \sup _{x \in B\left(\theta_{-} \omega\right)}\|x\|_{H}=0$ for all $\gamma>0$. A random variable $\omega \mapsto r(\omega) \in \mathbf{R}$ is said to be tempered with respect to $\left(\theta_{t}\right)_{t \in \mathbf{R}}$ if for a.e. $\omega \in \Omega_{0}, \lim _{t \rightarrow+\infty} \sup _{t \in \mathbf{R}} e^{-\gamma t} r\left(\theta_{-t} \omega\right)=$ 0 for all $\gamma>0$.

We consider a continuous random dynamical system (RDS) $\{\varphi(t, \omega)\}_{t \geq 0, \omega \in \Omega_{0}}$ over $\left(\Omega_{0}, \mathcal{F}, \mathbb{P},\left(\theta_{t}\right)_{t \in \mathbf{R}}\right)$ and $\boldsymbol{\Phi}(H)$ the set of all tempered random sets of $\boldsymbol{\Phi}(H)$.

Definition 2.3. A random set $\mathcal{K}$ is called an absorbing set in $\Phi(H)$ if for all $B \in \Phi(H)$ and a.e. $\omega \in \Omega_{0}$ there exists $t_{B}(\omega)>0$ such that

$$
\varphi\left(t, \theta_{-t} \omega\right) B\left(\theta_{-t} \omega\right) \subset \mathcal{K}(\omega) \quad \forall t \geq t_{B}(\omega)
$$


Definition 2.4. A random set $\mathscr{A}$ is called a global random $\Phi(H)$ attractor (pullback $\Phi(H)$ attractor) for $\{\varphi(t, \omega)\}_{t \geq 0, \omega \in \Omega_{0}}$ if the following hold:

(i) $\mathcal{A}$ is a random compact set, that is, $\omega \mapsto d(x, \mathcal{A}(\omega))$ is measurable for every $x \in H$ and $\mathcal{A}(\omega))$ is compact for a.e. $\omega \in \Omega_{0}$;

(ii) $\mathcal{A}$ is strictly invariant, that is, for $\omega \in \Omega_{0}$ and all $t \geq 0, \varphi(t, \omega) \mathcal{A}(\omega)=\mathcal{A}\left(\theta_{t} \omega\right)$;

(iii) $\mathcal{A}$ attracts all sets in $\Phi(H)$, that is, for all $B \in \Phi(H)$ and a.e. $\omega \in \Omega_{0}$, we have

$$
\lim _{t \rightarrow+\infty} d\left(\varphi\left(t, \theta_{-t} \omega\right) B\left(\theta_{-t} \omega\right), \mathcal{A}(\omega)\right)=0,
$$

where $d(X, Y)=\sup _{x \in X} \inf _{y \in Y}\|x-y\|_{H}$ is the Hausdorff semimetric $(X \subseteq H, Y \subseteq H)$.

Proposition 2.5 (see [11]). Suppose that

(a) there exists a random bounded absorbing set $K(\omega) \in \Phi\left(\ell^{2}\right), \omega \in \Omega_{0}$, such that for any $B(\omega) \in \Phi\left(\ell^{2}\right)$ and all $\omega \in \Omega_{0}$, there exists $T(\omega, B)>0$ yielding $\varphi\left(t, \theta_{-t} \omega, B\left(\theta_{-t} \omega\right)\right) \subset$ $K(\omega)$ for all $t \geq T(\omega, B)$;

(b) the RDS $\{\varphi(t, \omega)\}_{t>0, \omega \in \Omega_{0}}$ is random asymptotically null on $K(\omega)$, that is, for any $\epsilon>0$, there exist $T(\epsilon, \omega, K)>0$ and $I_{0}(\epsilon, \omega, K) \in \mathbb{N}$ such that

$$
\sup _{u \in K(\omega)} \sum_{|i|>I_{0}(\epsilon, \omega, K(\omega))}\left|\varphi\left(t, \theta_{-t} \omega, u\left(\theta_{-t} \omega\right)\right)_{i}\right|^{2} \leq \epsilon^{2}, \quad \forall t \geq T(\epsilon, \omega, K(\omega))
$$

Then the $\operatorname{RDS}\{\varphi(t, \omega, \cdot)\}_{t \geq 0, \omega \in \Omega_{0}}$ possesses a unique global random $\Phi(H)$ attractor given by

$$
\tilde{A}(\omega)=\bigcap_{\tau \geq T(\omega, K)} \overline{\bigcup_{t \geq \tau} \varphi\left(t, \theta_{-t} \omega, K\left(\theta_{-t} \omega\right)\right)} .
$$

\section{Existence of a Random Attractor}

In this section, we will derive the random attractor of the stochastic three-component reversible Gray-Scott lattice system (1.1) with a multiplicative white noise. follows:

For $x=\left(x_{i}\right)_{i \in \mathbb{Z}}$, we define $A, B, B^{*}$ to be linear operators from $\ell^{2}$ to $\ell^{2}$ for $i \in \mathbb{Z}$, as

$$
(A x)_{i}=-x_{i-1}+2 x_{i}-x_{i+1}, \quad(B x)_{i}=x_{i+1}-x_{i,} \quad\left(B^{*} x\right)_{i}=x_{i-1}-x_{i} .
$$

It is easy to show that $A=B B^{*}=B^{*} B,\left(B^{*} x, x^{\prime}\right)=\left(x, B x^{\prime}\right)$ for all $x, x^{\prime} \in \ell^{2}$, which implies that $(A x, x) \geq 0$. 
In the sequel, we rewrite the system (1.1) with initial values $\left(u_{0}, v_{0}, w_{0}\right):=$ $\left(u_{0 i}, v_{0 i}, w_{0 i}\right)_{i \in \mathbb{Z}} \in \ell^{2} \times \ell^{2} \times \ell^{2}=H$ as the following integral equations in $H$ for $t \geq 0, \omega \in \Omega$ :

$$
\begin{gathered}
u(t)=u_{0}+\int_{0}^{t}\left[-d_{1} A u(s)-(F+k) \mathrm{u}(s)+u^{2}(s) v(s)\right. \\
\left.-G u^{3}(s)+N w(s)\right] d s+\int_{0}^{t} u(s) \circ d W \\
v(t)=v_{0}+\int_{0}^{t}\left[-d_{2} A v(s)+F(1-v(s))-u^{2}(s) v(s)+G u^{3}(s)\right] d s+\int_{0}^{t} v(s) \circ d W, \\
w(t)=w_{0}+\int_{0}^{t}\left[-d_{3} A w(s)+k u(s)-(F+N) w(s)\right] d s+\int_{0}^{t} w(s) \circ d W
\end{gathered}
$$

where $W$ is a two-sided Brownian motion on the same probability space $(\Omega, \mathcal{F}, \mathbb{P})$. To prove that this system (3.2) generates a random dynamical system, we will transform it into a random differential equation system in $H$.

Before performing this transformation, we need to recall some properties of the Ornstein-Uhlenbeck processes. Let

$$
z\left(\theta_{t} \omega\right)=-\int_{-\infty}^{0} e^{\tau} \theta_{t} \omega(\tau) d \tau, \quad t \in \mathbf{R}, \omega \in \Omega
$$

We know that $z\left(\theta_{t} \omega\right)$ is an Ornstein-Uhlenbeck process on $\left(\Omega, \mathcal{F}, \mathbb{P},\left(\theta_{t}\right)_{t \in \mathbf{R}}\right)$ and solves the following one-dimensional stochastic differential equation (see [20] for details):

$$
z=-z d t+d w(t), \quad z(-\infty)=0, \quad \forall t \geq 0, \omega \in \Omega,
$$

where $w(t)(\omega)=w(t, \omega)=\omega(t)$ for $\omega \in \Omega, t \in \mathbf{R}$. In fact, we have the following.

Lemma 3.1 (see $[10,18]$ ). There exists a $\theta_{t}$-variant set $\widetilde{\Omega} \in \mathcal{F}$ of $\mathcal{C}_{0}(\mathbf{R}, \mathbf{R})$ of full $\mathbb{P}$ measure such that, for $\omega \in \widetilde{\Omega}$, one has

(i) the random variable $|z(\omega)|$ is tempered;

(ii) the mapping

$$
(t, \omega) \longmapsto z\left(\theta_{t} \omega\right)=-\int_{-\infty}^{0} e^{s} \omega(t+s) d s+\omega(t)
$$

is a stationary solution of Ornstein-Uhlenbeck equation (3.4) with continuous trajectories; (iii)

$$
\begin{gathered}
\lim _{t \rightarrow \pm \infty} \frac{\left|z\left(\theta_{t} \omega\right)\right|}{t}=0, \quad \lim _{t \rightarrow \pm \infty} \frac{1}{t} \int_{0}^{t} z\left(\theta_{s} \omega\right) d s=0, \\
\lim _{t \rightarrow \pm \infty} \frac{1}{t} \int_{0}^{t}\left|z\left(\theta_{s} \omega\right)\right| d s=\mathbb{E}|z|<\infty .
\end{gathered}
$$


Obviously, $e^{z\left(\theta_{t} \omega\right)} \mathbf{1} \mathbf{d}_{H}$ is clearly a homeomorphism in $H$, and the inverse operator $e^{-z\left(\theta_{t} \omega\right)} \mathbf{1} \mathbf{d}_{H}$ is well defined. It easily follows from (3.6) that $\left\|e^{z\left(\theta_{t} \omega\right)} \mathbf{1} \mathbf{d}_{H}\right\|_{H}$ and $\left\|e^{-z\left(\theta_{t} \omega\right)} \mathbf{1}_{H}\right\|_{H}$ have subexponential growth as $t \rightarrow \pm \infty$ for all $\omega \in \widetilde{\Omega}$, which implies that they are tempered. Since the mapping of $\theta$ on $\widetilde{\Omega}$ has the same properties as the original one if we choose the trace $\sigma$-algebra with respect to $\widetilde{\Omega}$ to be denoted also by $\mathcal{F}$, we can change our metric dynamical system with respect to $\widetilde{\Omega}$, and still denoted by the symbols $\left(\Omega, \mathcal{F}, \mathbb{P},\left(\theta_{t}\right)_{t \in \mathbf{R}}\right)$.

Let

$$
\tilde{g}(t, \omega)=e^{-z\left(\theta_{t} \omega\right)} \mathbf{1} \mathbf{d}_{H} g(t, \omega), \quad \omega \in \Omega,
$$

where $\tilde{g}(t, \omega)=(\widetilde{u}(t, \omega), \widetilde{v}(t, \omega), \widetilde{w}(t, \omega))$, and $g(t, \omega)=(u(t, \omega), v(t, \omega), w(t, \omega))$ is a solution of (3.2). Then system (1.1) can be written as the following random system with random coefficients but without white noise:

$$
\begin{gathered}
\frac{d \tilde{u}}{d t}=-d_{1} A \tilde{u}-\left(F+k-z\left(\theta_{t} \omega\right)\right) \tilde{u}+e^{2 z\left(\theta_{t} \omega\right)} \tilde{u}^{2} \widetilde{v}-G e^{2 z\left(\theta_{t} \omega\right)} \tilde{u}^{3}+N \widetilde{w}, \\
\frac{d \widetilde{v}}{d t}=-d_{2} A \widetilde{v}+F e^{-z\left(\theta_{t} \omega\right)}-\left(F-z\left(\theta_{t} \omega\right)\right) \widetilde{v}-e^{2 z\left(\theta_{t} \omega\right)} \tilde{u}^{2} \widetilde{v}+G e^{2 z\left(\theta_{t} \omega\right)} \tilde{u}^{3}, \\
\frac{d \widetilde{w}}{d t}=-d_{3} A \widetilde{w}+k \tilde{u}-\left(F+N-z\left(\theta_{t} \omega\right)\right) \tilde{w}
\end{gathered}
$$

and an initial condition

$$
\widetilde{g}_{0}(\omega)=(\widetilde{u}(0), \widetilde{v}(0), \widetilde{w}(0))=\left(\tilde{u}_{0}, \widetilde{v}_{0}, \widetilde{w}_{0}\right) \in H
$$

Now we establish the following result.

Theorem 3.2. Let $T>0$ and $\widetilde{g}_{0}(\omega) \in H$ be fixed. Then the following properties hold:

(i) for every $\omega \in \Omega$, system (3.9) admits a unique solution $\tilde{g}\left(\cdot, \omega, \tilde{g}_{0}\right) \in \mathcal{C}([0, T], H)$,

(ii) the solution $\widetilde{g}(t, \omega)$ of system (3.9) depends continuously on the initial data $\widetilde{g}_{0}$, that is, for each $\omega \in \Omega$, the mapping $\widetilde{g}_{0} \in H \mapsto \widetilde{g}\left(\cdot, \omega, \widetilde{g}_{0}\right) \in \mathcal{C}([0, T], H)$ is continuous.

Proof. (1) Denote

$$
\begin{gathered}
L(\tilde{g})=\left(\begin{array}{ccc}
-\left(d_{1} A+F+k\right) \tilde{u} & 0 & N \tilde{w} \\
0 & -\left(d_{2} A+F\right) \tilde{v} & 0 \\
k \tilde{u} & 0 & -\left(d_{3} A+F+N\right) \tilde{w}
\end{array}\right), \\
\Psi\left(\tilde{g}, \theta_{t} \omega\right)=\left(\begin{array}{c}
z\left(\theta_{t} \omega\right) \tilde{u}+e^{2 z\left(\theta_{t} \omega\right)} \tilde{u}^{2} \widetilde{v}-G e^{2 z\left(\theta_{t} \omega\right)} \tilde{u}^{3} \\
F e^{-z\left(\theta_{t} \omega\right)}+z\left(\theta_{t} \omega\right) \tilde{v}-e^{2 z\left(\theta_{t} \omega\right)} \tilde{u}^{2} \widetilde{v}+G e^{2 z\left(\theta_{t} \omega\right)} \tilde{u}^{3} \\
z\left(\theta_{t} \omega\right) \tilde{w}
\end{array}\right) .
\end{gathered}
$$

Then system (3.9) can be written as

$$
\frac{d \widetilde{g}}{d t}=L(\widetilde{g})+\Psi\left(\widetilde{g}, \theta_{t} \omega\right)
$$


Since $z\left(\theta_{t} \omega\right)$ is continuous with respect to $t$, define

$$
\Psi(\widetilde{g}, \omega)=\left(\begin{array}{c}
z(\omega) \tilde{u}+e^{2 z(\omega)} \tilde{u}^{2} \widetilde{v}-G e^{2 z(\omega)} \tilde{u}^{3} \\
F e^{-z(\omega)}+z(\omega) \widetilde{v}-e^{2 z(\omega)} \tilde{u}^{2} \widetilde{v}+G e^{2 z(\omega)} \tilde{u}^{3} \\
z(\omega) \widetilde{w}
\end{array}\right)
$$

then

$$
\begin{aligned}
\|\Psi(\tilde{g}, \omega)\|_{H} \leq & 2 F\left|e^{-z(\omega)}\right|+2\left(|z(\omega)|+\max \{1, G\} e^{2 z(\omega)}\right)\|\tilde{g}\|_{H} \\
& +3(1+G) e^{2 z(\omega)}\|\widetilde{g}\|_{H}^{2}
\end{aligned}
$$

For any $g=(u, v, w)=\left(u_{i}, v_{i}, w_{i}\right)_{i \in \mathbb{Z}}, \tilde{g}=(\tilde{u}, \tilde{v}, \tilde{w})=\left(\tilde{u}_{i}, \tilde{v}_{i}, \tilde{w}_{i}\right)_{i \in \mathbb{Z}} \in H$,

$$
\|\Psi(g, \omega)-\Psi(\tilde{g}, \omega)\|_{H} \leq 2\left(\| z(\omega) \mid+3(1+G) e^{2 z(\omega)}\left(\|g\|_{H}^{2}+\|\tilde{g}\|_{H}^{2}\right)\right)\|g-\tilde{g}\|_{H}
$$

For any bounded set $D \in H$ with $\sup _{g \in D}\|g\|_{H} \leq r$, define a random variable $\rho_{D}(\omega)$ by

$$
\rho_{D}(\omega)=2\left(|z(\omega)|+3(1+G) e^{2 z(\omega)} r+\max \{1, G\} e^{2 z(\omega)}\right) r+2 F\left|e^{-z(\omega)}\right| \geq 0
$$

Then

$$
\begin{aligned}
\int_{\tau}^{\tau+1} \rho_{D}\left(\theta_{t} \omega\right) d t & =2 \int_{\tau}^{\tau+1}\left(\left(\left|z\left(\theta_{t} \omega\right)\right|+3(1+G) e^{2 z\left(\theta_{t} \omega\right)} r+\max \{1, G\} e^{2 z\left(\theta_{t} \omega\right)}\right) r+F e^{-z\left(\theta_{t} \omega\right)}\right) d t \\
& <\infty, \quad \forall \tau \in \mathbf{R},
\end{aligned}
$$

and, for any $\bar{g}, g, \tilde{g} \in D$, we have

$$
\|\Psi(\bar{g}, \omega)\|_{H} \leq \rho_{D}(\omega), \quad\|\Psi(g, \omega)-\Psi(\tilde{g}, \omega)\|_{H} \leq Q_{D}(\omega)\|g-\tilde{g}\|_{H} .
$$

Then we obtain that $L(\widetilde{g})+\Psi\left(\widetilde{g}, \theta_{t} \omega\right)$ is locally Lipschitz in $\widetilde{g}$ from $H$ to $H$. By Proposition 2.1.1 in [19], problem (3.12) possesses a unique local solution $\tilde{g}\left(\cdot, \omega, \tilde{g}_{0}\right) \in \mathcal{C}^{1}\left(\left(0, T_{\max }\right), H\right)$, where $\left(0, T_{\max }\right)$ is the maximal interval of existence of the solution of (3.12). Now, we will show that the local solution is a global one. Define

$$
\tilde{r}(t)=\frac{N}{k} \tilde{w}(t), \quad \mu=\frac{k}{N},
$$


then system (3.9) can be written as

$$
\begin{gathered}
\frac{d \tilde{u}}{d t}=-d_{1} A \tilde{u}-\left(F+k-z\left(\theta_{t} \omega\right)\right) \tilde{u}+e^{2 z\left(\theta_{t} \omega\right)} \tilde{u}^{2} \widetilde{v}-G e^{2 z\left(\theta_{t} \omega\right)} \tilde{u}^{3}+k \tilde{r}, \\
\frac{d \tilde{v}}{d t}=-d_{2} A \tilde{v}+F e^{-z\left(\theta_{t} \omega\right)}-\left(F-z\left(\theta_{t} \omega\right)\right) \tilde{v}-e^{2 z\left(\theta_{t} \omega\right)} \tilde{u}^{2} \widetilde{v}+G e^{2 z\left(\theta_{t} \omega\right)} \tilde{u}^{3}, \\
\mu \frac{d \tilde{r}}{d t}=-\mu d_{3} A \tilde{r}+k \tilde{u}-\left(\mu F+k-z\left(\theta_{t} \omega\right)\right) \tilde{r} .
\end{gathered}
$$

Taking the inner products of (3.20) with $G \tilde{u}(t), \widetilde{v}(t)$ and $G \tilde{w}(t)$, respectively, and adding up the resulting equalities, we get

$$
\begin{gathered}
\frac{1}{2} \frac{d}{d t}\left(G\|\tilde{u}\|^{2}+\|\tilde{v}\|^{2}+\mu G\|\tilde{r}\|^{2}\right)+G F\|\tilde{u}\|^{2}+\frac{F}{2}\|\widetilde{v}\|^{2}+\mu G F\|\tilde{r}\|^{2} \\
\leq z\left(\theta_{t} \omega\right)\left(G\|\tilde{u}\|^{2}+\|\tilde{v}\|^{2}+G\|\tilde{r}\|^{2}\right)+\frac{F}{2} e^{-2 z\left(\theta_{t} \omega\right)},
\end{gathered}
$$

which implies that

$$
\begin{gathered}
\frac{d}{d t}\left(G\|\tilde{u}\|^{2}+\|\tilde{v}\|^{2}+\frac{G}{\mu}\|\tilde{w}\|^{2}\right)+2 G F\|\tilde{u}\|^{2}+F\|\tilde{v}\|^{2}+\frac{2 G F}{\mu}\|\tilde{w}\|^{2} \\
\leq 2 z\left(\theta_{t} \omega\right)\left(G\|\tilde{u}\|^{2}+\|\tilde{v}\|^{2}+\frac{G}{\mu^{2}}\|\tilde{w}\|^{2}\right)+F e^{-2 z\left(\theta_{t} \omega\right)}
\end{gathered}
$$

Setting

$$
\lambda=\frac{F \min \{1,2 G, 2 G / \mu\}}{\max \{1, G, G / \mu\}}, \quad C_{1}=\frac{2 \max \left\{1, G, G / \mu^{2}\right\}}{\min \{1, G, G / \mu\}}, \quad C_{2}=\frac{F}{\min \{1, G, G / \mu\}},
$$

then (3.22) yields

$$
\frac{d}{d t}\|\widetilde{g}(t)\|_{H}^{2}+\left(\lambda-C_{1} z\left(\theta_{t} \omega\right)\right)\|\tilde{g}(t)\|_{H}^{2} \leq C_{2} e^{-2 z\left(\theta_{t} \omega\right)} .
$$

Applying Gronwall's lemma to (3.24), we obtain that, for $t \geq 0$,

$$
\begin{aligned}
\left\|\tilde{g}\left(t, \omega, \tilde{g}_{0}(\omega)\right)\right\|_{H}^{2} \leq & e^{-\lambda t+C_{1} \int_{0}^{t} z\left(\theta_{s} \omega\right) d s}\left\|\tilde{g}_{0}(\omega)\right\|_{H}^{2} \\
& +C_{2} e^{-\lambda t+C_{1} \int_{0}^{t} z\left(\theta_{s} \omega\right) d s} \int_{0}^{t} e^{-2 z\left(\theta_{s} \omega\right)+\lambda s+C_{1} \int_{s}^{0} z\left(\theta_{\tau} \omega\right) d \tau} d s .
\end{aligned}
$$


Denoting

$$
\begin{gathered}
\alpha(\omega)=C_{1} \int_{0}^{T}\left|z\left(\theta_{s} \omega\right)\right| d s \\
\beta(\omega)=C_{2} \max _{t \in[0, T]}\left(e^{-\lambda t+C_{1} \int_{0}^{t} z\left(\theta_{s} \omega\right) d s} \int_{0}^{t} e^{-2 z\left(\theta_{s} \omega\right)+\lambda s+C_{1} \int_{s}^{0} z\left(\theta_{\tau} \omega\right) d \tau} d s\right),
\end{gathered}
$$

we get

$$
\left\|\tilde{g}\left(t, \omega, \tilde{g}_{0}(\omega)\right)\right\|_{H}^{2} \leq\left\|\tilde{g}_{0}(\omega)\right\|_{H}^{2} e^{\alpha(\omega)}+\beta(\omega),
$$

which implies that the solution $\widetilde{g}$ is defined in any interval $[0, T]$.

(2) Let $g_{0}, \widetilde{g}_{0} \in H$ and

$$
X(t):=g\left(t, \omega, g_{0}\right), \quad Y(t):=\tilde{g}\left(t, \omega, \widetilde{g}_{0}\right)
$$

be the corresponding solutions of (3.12). Then, denoting $Z(t)=\left(Z_{1}, Z_{2}, Z_{3}\right)=X(t)-Y(t)=$ $(u-\tilde{u}, v-\widetilde{v}, w-\tilde{w})$, we have

$$
\begin{gathered}
\frac{d Z_{1}}{d t}=-d_{1} A Z_{1}-\left(F+k-z\left(\theta_{t} \omega\right)\right) Z_{1}+N Z_{3}+e^{2 z\left(\theta_{t} \omega\right)}\left(u^{2} v-\tilde{u}^{2} \tilde{v}\right)-G e^{2 z\left(\theta_{t} \omega\right)}\left(u^{3}-\tilde{u}^{3}\right) \\
\frac{d Z_{2}}{d t}=-d_{2} A Z_{2}-\left(F-z\left(\theta_{t} \omega\right)\right) Z_{2}-e^{2 z\left(\theta_{t} \omega\right)}\left(u^{2} v-\tilde{u}^{2} \tilde{v}\right)+G e^{2 z\left(\theta_{t} \omega\right)}\left(u^{3}-\tilde{u}^{3}\right) \\
\frac{d Z_{3}}{d t}=-d_{3} A Z_{3}+k Z_{1}-\left(F+N-z\left(\theta_{t} \omega\right)\right) Z_{3} .
\end{gathered}
$$

Taking the inner product of (3.30) with $Z_{1}, Z_{2}$ and $Z_{3}$, respectively, it yields

$$
\begin{aligned}
\frac{d}{d t}\left(\left\|Z_{1}\right\|^{2}\right. & \left.+\left\|Z_{2}\right\|^{2}+\left\|Z_{3}\right\|^{2}\right) \\
\leq & 2 z\left(\theta_{t} \omega\right)\left(\left\|Z_{1}\right\|^{2}+\left\|Z_{2}\right\|^{2}+\left\|Z_{3}\right\|^{2}\right)+\frac{N}{2}\left\|Z_{1}\right\|^{2}+\frac{k}{2}\left\|Z_{3}\right\|^{2} \\
& +2 e^{2 z\left(\theta_{t} \omega\right)}\left(u^{2} v-\tilde{u}^{2} \widetilde{v}, Z_{1}-Z_{2}\right)-2 G e^{2 z\left(\theta_{t} \omega\right)}\left(u^{3}-\tilde{u}^{3}, Z_{1}-Z_{2}\right)
\end{aligned}
$$

Due to (3.28), we have

$$
\begin{gathered}
e^{2 z\left(\theta_{t} \omega\right)}\left(u^{2} v-\tilde{u}^{2} \tilde{v}, Z_{1}-Z_{2}\right)-G e^{2 z\left(\theta_{t} \omega\right)}\left(u^{3}-\tilde{u}^{3}, Z_{1}-Z_{2}\right) \\
\leq 2(1+G) e^{2 z\left(\theta_{t} \omega\right)}\left(\|g\|_{H}^{2}+\|\tilde{g}\|_{H}^{2}\right)\left(\left\|Z_{1}\right\|^{2}+\left\|Z_{2}\right\|^{2}\right) \\
\leq 4(1+G) e^{2 z\left(\theta_{t} \omega\right)}\|\tilde{g}\|_{H}^{2}\left(\left\|Z_{1}\right\|^{2}+\left\|Z_{2}\right\|^{2}+\left\|Z_{3}\right\|^{2}\right) .
\end{gathered}
$$


Denoting $\delta=(1 / 2) \max \{N, k\}$, we obtain that

$$
\begin{aligned}
& \frac{d}{d t}\left(\left\|Z_{1}\right\|^{2}+\left\|Z_{2}\right\|^{2}+\left\|Z_{3}\right\|^{2}\right) \\
& \quad \leq\left(\delta+z\left(\theta_{t} \omega\right)+8(1+G) e^{2 z\left(\theta_{t} \omega\right)}\|\tilde{g}\|_{H}^{2}\right)\left(\left\|Z_{1}\right\|^{2}+\left\|Z_{2}\right\|^{2}+\left\|Z_{3}\right\|^{2}\right) .
\end{aligned}
$$

By Gronwall's lemma, for $t \in[0, T]$, we have

$$
\left\|Z_{1}\right\|^{2}+\left\|Z_{2}\right\|^{2}+\left\|Z_{3}\right\|^{2} \leq\left(\left\|Z_{1}(0)\right\|^{2}+\left\|Z_{2}(0)\right\|^{2}+\| Z_{3}(0)\right) e^{\delta^{\prime} t+8(1+G) \int_{0}^{t} e^{2 z}\left(\theta_{s} \omega\right)\left\|\tilde{g}\left(s, \omega, \tilde{g}_{0}(\omega)\right)\right\|_{H}^{2} d s},
$$

where $\delta^{\prime}=\delta+\max _{t \in[0, T]}\left|z\left(\theta_{t} \omega\right)\right|$. According to (3.26), for $t \in[0, T]$,

$$
\begin{aligned}
\int_{0}^{t} e^{2 z\left(\theta_{s} \omega\right)}\left\|\widetilde{g}\left(s, \omega, \widetilde{g}_{0}(\omega)\right)\right\|_{H}^{2} d s \\
\leq\left\|\widetilde{g}_{0}(\omega)\right\|_{H}^{2} \int_{0}^{t} e^{2 z\left(\theta_{s} \omega\right)+C_{1} \int_{0}^{s} z\left(\theta_{\tau} \omega\right) d \tau} d s \\
\quad+C_{2} \int_{0}^{t} e^{2 z\left(\theta_{s} \omega\right)+C_{1} \int_{0}^{s} z\left(\theta_{\tau} \omega\right) d \tau} \int_{0}^{s} e^{-2 z\left(\theta_{\tau} \omega\right)-C_{1} \int_{0}^{t} z\left(\theta_{s} \omega\right) d s} d \tau d s \\
\quad:=\left\|\widetilde{g}_{0}(\omega)\right\|_{H}^{2} \int_{0}^{t} \xi\left(\theta_{s} \omega\right) d s+C_{2} \int_{0}^{t} \xi\left(\theta_{s} \omega\right) \int_{0}^{s} \xi^{-1}\left(\theta_{\tau} \omega\right) d \tau d s,
\end{aligned}
$$

where $\xi\left(\theta_{t} \omega\right)=e^{2 z\left(\theta_{t} \omega\right)+C_{1} \int_{0}^{t} z\left(\theta_{\tau} \omega\right) d \tau}$. Obviously, $t \mapsto \ln \xi\left(\theta_{t} \omega\right)$ is continuous $\mathbb{P}$-a.s. Also, we have $\lim _{t \rightarrow+\infty}\left(\ln \xi\left(\theta_{t} \omega\right) / t\right)=0$, which implies that $\xi\left(\theta_{t} \omega\right)$ is a tempered random variable. Then by Proposition 4.3.3, [18], for given $\varepsilon>0$, there is an $\varepsilon$-slowly varying random variable $R(\omega)$ for which

$$
e^{-\varepsilon|t|} R(\omega) \leq \xi\left(\theta_{t} \omega\right) \leq e^{\varepsilon|t|} R(\omega), \quad \forall t \in \mathbf{R}, \omega \in \Omega,
$$

where $R(\omega), \omega \in \Omega$ satisfies

$$
e^{-\varepsilon|t|} R(\omega) \leq R\left(\theta_{t} \omega\right) \leq e^{\varepsilon|t|} R(\omega) \quad \forall t \in \mathbf{R}, \omega \in \Omega
$$

Combining with (3.35) and (3.36), we easily conclude that for $t \in[0, T]$,

$$
\int_{0}^{t} e^{2 z\left(\theta_{s} \omega\right)}\left\|\tilde{g}\left(s, \omega, \widetilde{g}_{0}(\omega)\right)\right\|_{H}^{2} d s \leq \frac{\left\|\widetilde{g}_{0}(\omega)\right\|_{H}^{2}}{\varepsilon} R(\omega) e^{\varepsilon T}+\frac{C_{2}}{2 \varepsilon^{2}} e^{2 \varepsilon T},
$$


which implies

$$
\sup _{t \in[0, T]}\|X(t)-Y(t)\|^{2} \leq\left(\left\|u_{0}-\tilde{u}_{0}\right\|^{2}+\left\|v_{0}-\widetilde{v}_{0}\right\|^{2}+\left\|w_{0}-\widetilde{w}_{0}\right\|^{2}\right) e^{\delta^{\prime \prime} T}
$$

where $\delta^{\prime \prime}=\delta^{\prime}+8(1+\mathrm{G})\left(\left(\left\|\widetilde{g}_{0}(\omega)\right\|_{H}^{2} / \varepsilon T\right) R(\omega) e^{\varepsilon T}+\left(C_{2} / 2 \varepsilon^{2} T\right) e^{2 \varepsilon T}\right)<\infty$. If $u_{0}=\widetilde{u}_{0}, v_{0}=\widetilde{v}_{0}$ and $w_{0}=\widetilde{w}_{0}$, then the above inequality shows the uniqueness and continuous dependence on the initial data of the solution of (3.12). So the both results of the theorem hold.

Theorem 3.3. System (3.12) generates a continuous random dynamical system $(\varphi(t))_{t \geq 0}$ over $(\Omega$, $\left.\mathcal{F}, \mathbb{P},\left(\theta_{t}\right)_{t \in \mathbf{R}}\right)$, where $\varphi\left(t, \omega, g_{0}\right)=\tilde{g}\left(t, \omega, g_{0}\right)$ for $g_{0} \in H, t \geq 0$ and for all $\omega \in \Omega$. Moreover, if one defines $\varphi$ by

$$
\psi\left(t, \omega, g_{0}\right)=e^{z\left(\theta_{t} \omega\right)} \varphi\left(t, \omega, e^{-z(\omega)} g_{0}\right)
$$

for $g_{0} \in H, t \geq 0$ and for all $\omega \in \Omega$, then $\psi$ is another random dynamical system for which the process $(\omega, t) \mapsto \psi\left(t, \omega, g_{0}\right)$ solves (3.2) for any initial condition $g_{0} \in H$.

Proof. The fact that $\varphi$ is continuous random dynamical system follows from Theorem 3.2. The measurability of $\psi$ follows from the properties of the transformation (see $[18,19])$. It follows directly the other statements.

Note that the two random dynamical systems are equivalent. It is easy to check that $\psi$ has a random attractor provided $\varphi$ possesses a random attractor. Then, we only need to consider the random dynamical system $\varphi$.

Now, we are in the position to study the existence of tempered random bounded absorbing set and global random attractor for the $\operatorname{RDS} \varphi$ in $H$.

Lemma 3.4. There exists a random bounded ball $K(\omega) \in \boldsymbol{\Phi}(H)$ centered at 0 with random radius $\rho(\omega)>0$ such that $K(\omega) \in \Phi(H)$ is a random absorbing set for $\varphi$ in $\Phi(H)$; that is, for any $B(\omega) \in$ $\Phi(H)$ and $\omega \in \Omega$, there exists $T_{B}(\omega)>0$ yielding $\varphi\left(t, \theta_{-t} \omega, B\left(\theta_{-t} \omega\right)\right) \subset K(\omega)$, for all $t \geq T_{B}(\omega)$.

Proof. By substituting $\omega$ by $\theta_{-t} \omega$ in (3.26), we have

$$
\begin{aligned}
& \left\|\tilde{g}\left(t, \theta_{-t} \omega, \tilde{g}_{0}\left(\theta_{-t} \omega\right)\right)\right\|_{H}^{2} \\
& \quad \leq e^{-\lambda t+C_{1} \int_{0}^{t} z\left(\theta_{s-t} \omega\right) d s}\left\|\tilde{g}_{0}\left(\theta_{-t} \omega\right)\right\|_{H}^{2}+C_{2} e^{-\lambda t+C_{1} \int_{0}^{t} z\left(\theta_{s-t} \omega\right) d s} \int_{0}^{t} e^{-2 z\left(\theta_{s-t} \omega\right)+\lambda s+C_{1} \int_{s}^{0} z\left(\theta_{\tau-t} \omega\right) d \tau} d s \\
& \quad \leq e^{-\lambda t+C_{1} \int_{0}^{t} z\left(\theta_{s-t} \omega\right) d s}\left\|\tilde{g}_{0}\left(\theta_{-t} \omega\right)\right\|_{H}^{2}+C_{2} \int_{-t}^{0} e^{-2 z\left(\theta_{s} \omega\right)+\lambda(s-t)+C_{1} \int_{s}^{t} z\left(\theta_{\tau} \omega\right) d \tau} d s \\
& \quad \leq e^{-\lambda t+C_{1} \int_{-t}^{0} z\left(\theta_{s} \omega\right) d s}\left\|\tilde{g}_{0}\left(\theta_{-t} \omega\right)\right\|_{H}^{2}+C_{2} \int_{-t}^{0} e^{-2 z\left(\theta_{s} \omega\right)+\lambda s+C_{1} \int_{s}^{0} z\left(\theta_{\tau} \omega\right) d \tau} d s \\
& \quad \leq e^{-\lambda t+C_{1} \int_{-t}^{0} z\left(\theta_{s} \omega\right) d s}\left\|\tilde{g}_{0}\left(\theta_{-t} \omega\right)\right\|_{H}^{2}+C_{2} \int_{-\infty}^{0} e^{-2 z\left(\theta_{s} \omega\right)+\lambda s+C_{1} \int_{s}^{0} z\left(\theta_{\tau} \omega\right) d \tau} d s .
\end{aligned}
$$


By the properties of the Ornstein-Uhlenbeck process,

$$
\int_{-\infty}^{0} e^{-2 z\left(\theta_{s} \omega\right)+\lambda s+C_{1} \int_{s}^{0} z\left(\theta_{\tau} \omega\right) d \tau} d s<+\infty
$$

Notice that $\{B(\omega)\} \in \Phi(H)$ is tempered, then for $\tilde{g}_{0}(\omega) \in B\left(\theta_{-t} \omega\right)$,

$$
\lim _{t \rightarrow+\infty} e^{-\lambda t+C_{1} \int_{-t}^{0} z\left(\theta_{s} \omega\right) d s}\left\|\widetilde{g}_{0}\left(\theta_{-t} \omega\right)\right\|_{H}^{2}=0 .
$$

We can choose

$$
\rho^{2}(\omega)=1+C_{2} \int_{-\infty}^{0} e^{-2 z\left(\theta_{s} \omega\right)+\lambda s+C_{1} \int_{s}^{0} z\left(\theta_{\tau} \omega\right) d \tau} d s
$$

then $K(\omega)$ is a random absorbing set for $\varphi$ in $\Phi(H)$, and $K(\omega) \in \Phi(H)$. Here, we remain only to check that

$$
\lim _{t \rightarrow+\infty} e^{-\gamma t} \rho^{2}\left(\theta_{-t} \omega\right)=0
$$

Indeed, obviously we have

$$
\begin{aligned}
e^{-\gamma t} \rho^{2}\left(\theta_{-t} \omega\right) & =e^{-\gamma t}+C_{2} e^{-\gamma t} \int_{-\infty}^{0} e^{-2 z\left(\theta_{s-t} \omega\right)+\lambda s+C_{1} \int_{s}^{0} z\left(\theta_{\tau-t} \omega\right) d \tau} d s \\
& =\underbrace{e^{-\gamma t}}_{\rightarrow 0 \text { as } t \rightarrow+\infty}+\underbrace{C_{2} e^{-\gamma t} \int_{-\infty}^{-t} e^{-2 z\left(\theta_{s} \omega\right)+\lambda s+C_{1} \int_{s}^{-t} z\left(\theta_{\tau} \omega\right) d \tau} d s}_{\rightarrow 0 \text { as } t \rightarrow+\infty} .
\end{aligned}
$$

Lemma 3.5. The RDS $\{\varphi(t, \omega, \cdot)\}_{t \geq 0, \omega \in \Omega}$ generated by (3.9) is random asymptotically null on $K(\omega)$; that is, for any $\epsilon>0$, there exist $T(\epsilon, \omega, K)>0, \forall t \geq T(\epsilon, \omega, K(\omega))$, and $M(\epsilon, \omega, K(\omega)) \in \mathbb{N}$ such that

$$
\sup _{\varphi_{0} \in K(\omega)} \sum_{|i|>M(\epsilon, \omega, K(\omega))}\left|\varphi\left(t, \theta_{-t} \omega, \varphi_{0}\left(\theta_{-t} \omega\right)\right)_{i}\right|_{H}^{2} \leq \epsilon^{2}
$$

Proof. Choose a smooth cut-off function satisfying $0 \leq \rho(s) \leq 1$ for $s \in \mathbb{R}^{+}$and $\rho(s)=0$ for $0 \leq s \leq 1, \rho(s)=1$ for $s \geq 2$. Suppose there exists a constant $c$ such that $\left|\rho^{\prime}(s)\right| \leq c$ for $s \in \mathbb{R}^{+}$.

Set $x=\left(\rho(|i| / M) \tilde{u}_{i}\right)_{i \in \mathbb{Z}}, y=\left(\rho(|i| / M) \tilde{v}_{i}\right)_{i \in \mathbb{Z}}$, and $z=\left(\rho(|i| / M) \tilde{w}_{i}\right)_{i \in \mathbb{Z}}$. By taking the inner product of (3.20) with $G x, y$, and $G z$, respectively, we get

$$
\begin{aligned}
\frac{G}{2} \frac{d}{d t} \sum_{i \in \mathbb{Z}} \rho\left(\frac{|i|}{M}\right)\left|\tilde{u}_{i}\right|^{2}= & -G d_{1}(A \tilde{u}, x)-G\left(F+k-z\left(\theta_{t} \omega\right)\right) \sum_{i \in \mathbb{Z}} \rho\left(\frac{|i|}{M}\right)\left|\tilde{u}_{i}\right|^{2} \\
& +k G(\tilde{r}, x)+G e^{2 z\left(\theta_{t} \omega\right)}\left(\tilde{u}^{2} \widetilde{v}, x\right)-G^{2} e^{2 z\left(\theta_{t} \omega\right)}\left(\tilde{u}^{3}, x\right),
\end{aligned}
$$


Discrete Dynamics in Nature and Society

$$
\begin{aligned}
\frac{1}{2} \frac{d}{d t} \sum_{i \in \mathbb{Z}} \rho\left(\frac{|i|}{M}\right)\left|\widetilde{v}_{i}\right|^{2}= & -d_{2}(A \widetilde{v}, y)-\left(F-z\left(\theta_{t} \omega\right)\right) \sum_{i \in \mathbb{Z}} \rho\left(\frac{|i|}{M}\right)\left|\widetilde{v}_{i}\right|^{2} \\
& -G e^{2 z\left(\theta_{t} \omega\right)}\left(\tilde{u}^{2} \widetilde{v}, y\right)+G^{2} e^{2 z\left(\theta_{t} \omega\right)}\left(\tilde{u}^{3}, y\right)+\left(e^{-z\left(\theta_{t} \omega\right)}, y\right), \\
\frac{\mu G}{2} \frac{d}{d t} \sum_{i \in \mathbb{Z}} \rho\left(\frac{|i|}{M}\right)\left|\tilde{r}_{i}\right|^{2}= & -\mu G d_{3}(A \widetilde{r}, z)-G\left(\mu F+k-z\left(\theta_{t} \omega\right)\right) \sum_{i \in \mathbb{Z}} \rho\left(\frac{|i|}{M}\right)\left|\tilde{r}_{i}\right|^{2} \\
& +k G(\tilde{u}, z) .
\end{aligned}
$$

Due to $[9,10]$, we have

$$
-(A \tilde{u}, x) \leq \frac{2 c\|\tilde{u}\|^{2}}{M}, \quad-(A \tilde{v}, y) \leq \frac{2 c\|\tilde{v}\|^{2}}{M}, \quad-(A \tilde{r}, z) \leq \frac{2 c\|\tilde{r}\|^{2}}{M} .
$$

Combining with (3.48) to (3.49), we obtain

$$
\begin{gathered}
\frac{1}{2} \frac{d}{d t} \sum_{i \in \mathbb{Z}} \rho\left(\frac{|i|}{M}\right)\left(G\left|\tilde{u}_{i}\right|^{2}+\left|\tilde{v}_{i}\right|^{2}+\mu G\left|\tilde{r}_{i}\right|^{2}\right)+G\left(F-z\left(\theta_{t} \omega\right)\right) \sum_{i \in \mathbb{Z}} \rho\left(\frac{|i|}{M}\right)\left|\tilde{u}_{i}\right|^{2} \\
+\left(\frac{F}{2}-z\left(\theta_{t} \omega\right)\right) \sum_{i \in \mathbb{Z}} \rho\left(\frac{|i|}{M}\right)\left|\widetilde{v}_{i}\right|^{2}+G\left(\mu F-z\left(\theta_{t} \omega\right)\right) \sum_{i \in \mathbb{Z}} \rho\left(\frac{|i|}{M}\right)\left|\tilde{r}_{i}\right|^{2} \\
\leq \frac{2 c}{M}\left(G d_{1}\|\tilde{u}\|^{2}+d_{2}\|\widetilde{v}\|^{2}+\mu G d_{3}\|\tilde{r}\|^{2}\right)+\frac{F}{2} e^{-2 z\left(\theta_{t} \omega\right)},
\end{gathered}
$$

that is,

$$
\begin{aligned}
& \frac{d}{d t} \sum_{i \in \mathbb{Z}} \rho\left(\frac{|i|}{M}\right)\left(G\left|\tilde{u}_{i}\right|^{2}+\left|\tilde{v}_{i}\right|^{2}+\frac{G}{\mu}\left|\tilde{w}_{i}\right|^{2}\right)+2 G F \sum_{i \in \mathbb{Z}} \rho\left(\frac{|i|}{M}\right)\left|\tilde{u}_{i}\right|^{2} \\
& \quad+F \sum_{i \in \mathbb{Z}} \rho\left(\frac{|i|}{M}\right)\left|\tilde{v}_{i}\right|^{2}+\frac{2 G F}{\mu} \sum_{i \in \mathbb{Z}} \rho\left(\frac{|i|}{M}\right)\left|\tilde{w}_{i}\right|^{2} \\
& \leq 2 z\left(\theta_{t} \omega\right) \sum_{i \in \mathbb{Z}} \rho\left(\frac{|i|}{M}\right)\left(G\left|\tilde{u}_{i}\right|^{2}+\left|\tilde{v}_{i}\right|^{2}+\frac{G}{\mu^{2}}\left|\tilde{w}_{i}\right|^{2}\right) \\
& \quad+\frac{4 c}{M}\left(G d_{1}\|\tilde{u}\|^{2}+d_{2}\|\tilde{v}\|^{2}+\frac{G}{\mu} d_{3}\|\tilde{w}\|^{2}\right)+F e^{-2 z\left(\theta_{t} \omega\right)} .
\end{aligned}
$$

Denote

$$
C_{3}=2 c \max \left\{d_{1}, d_{2}, d_{3}\right\} C_{1},
$$


then (3.51) yields

$$
\frac{d}{d t} \sum_{i \in \mathbb{Z}} \rho\left(\frac{|i|}{M}\right)\left|\tilde{g}_{i}\right|_{H}^{2}+\left(\lambda-C_{1} z\left(\theta_{t} \omega\right)\right) \sum_{i \in \mathbb{Z}} \rho\left(\frac{|i|}{M}\right)\left|\tilde{g}_{i}\right|_{H}^{2} \leq \frac{C_{3}}{M}\|\tilde{g}\|_{H}^{2}+C_{2} e^{-2 z\left(\theta_{t} \omega\right)}
$$

By using Gronwall's lemma, for $t \geq T_{K}=T_{K}(\omega)$, we have

$$
\begin{aligned}
\sum_{i \in \mathbb{Z}} \rho\left(\frac{|i|}{M}\right)\left|\tilde{g}_{i}\left(t, \omega, \tilde{g}_{0}(\omega)\right)\right|_{H}^{2} \\
\leq e^{-\lambda\left(t-T_{K}\right)+C_{1} \int_{T_{k}}^{t} z\left(\theta_{s} \omega\right) d s} \sum_{i \in \mathbb{Z}} \rho\left(\frac{|i|}{M}\right)\left|\widetilde{g}_{i}\left(T_{k}, \omega, \widetilde{g}_{0}(\omega)\right)\right|_{H}^{2} \\
\quad+\frac{C_{3}}{M} \int_{T_{k}}^{t} e^{-\lambda(t-s)+C_{1} \int_{s}^{t} z\left(\theta_{\tau} \omega\right) d \tau}\left\|\tilde{g}\left(s, \omega, \widetilde{g}_{0}(\omega)\right)\right\|_{H}^{2} d s \\
\quad+C_{2} \int_{T_{k}}^{t} e^{-\lambda(t-s)-2 z\left(\theta_{s} \omega\right)+C_{1} \int_{s}^{t} z\left(\theta_{\tau} \omega\right) d \tau} d s .
\end{aligned}
$$

Replace $\omega$ by $\theta_{-t} \omega$. We then estimate each term on the right-hand side of (3.54). From (3.26) with $t$ replaced by $T_{K}$ and $\omega$ by $\theta_{-t} \omega$, respectively, it then follows that

$$
\begin{aligned}
& e^{-\lambda\left(t-T_{K}\right)+C_{1} \int_{T_{k}}^{t} z\left(\theta_{s-t} \omega\right) d s} \sum_{i \in \mathbb{Z}} \rho\left(\frac{|i|}{M}\right)\left|\widetilde{g}_{i}\left(T_{k}, \theta_{-t} \omega, \widetilde{g}_{0}\left(\theta_{-t} \omega\right)\right)\right|_{H}^{2} \\
& \quad \leq e^{-\lambda t+C_{1} \int_{0}^{t} z\left(\theta_{s-t} \omega\right) d s}\left\|\widetilde{g}_{0}(\omega)\right\|_{H}^{2}+C_{2} \int_{0}^{T_{K}} e^{-2 z\left(\theta_{s-t} \omega\right)+\lambda(s-t)+C_{1} \int_{s}^{t} z\left(\theta_{\tau-t} \omega\right) d \tau} d s \\
& \leq e^{-\lambda t+C_{1} \int_{-t}^{0} z\left(\theta_{s} \omega\right) d s}\left\|\widetilde{g}_{0}(\omega)\right\|_{H}^{2}+C_{2} \int_{-t}^{T_{K}-t} e^{-2 z\left(\theta_{s} \omega\right)+\lambda s+C_{1} \int_{s}^{0} z\left(\theta_{\tau} \omega\right) d \tau} d s .
\end{aligned}
$$

Hence, by using (3.6), there is a $T_{1}(\epsilon, \omega, K(\omega))>T_{K}(\omega)$, such that if $t>T_{1}(\epsilon, \omega, K(\omega))$,

$$
e^{-\lambda\left(t-T_{K}\right)+C_{1} \int_{T_{k}}^{t} z\left(\theta_{s-t} \omega\right) d s} \sum_{i \in \mathbb{Z}} \rho\left(\frac{|i|}{M}\right)\left|\tilde{g}_{i}\left(T_{k}, \theta_{-t} \omega, \tilde{g}_{0}\left(\theta_{-t} \omega\right)\right)\right|_{H}^{2}<\frac{\epsilon^{2}}{3}
$$


Next, we estimate

$$
\begin{aligned}
& \frac{C_{3}}{M} \int_{T_{k}}^{t} e^{-\lambda(t-s)+C_{1} \int_{s}^{t} z\left(\theta_{\tau-t} \omega\right) d \tau}\left\|\tilde{g}\left(s, \theta_{-t} \omega, \widetilde{g}_{0}\left(\theta_{-t} \omega\right)\right)\right\|_{H}^{2} d s \\
& \quad \leq \frac{C_{3}}{M}\left(t-T_{K}\right) e^{-\lambda t+C_{1} \int_{0}^{t} z\left(\theta_{r-t} \omega\right) d r}\left\|\widetilde{g}_{0}(\omega)\right\|_{H}^{2}+\frac{C_{2} C_{3}}{M} \int_{T_{K}}^{t} \int_{0}^{s} e^{-2 z\left(\theta_{\tau-t} \omega\right)-\lambda(t-\tau)+C_{1} \int_{\tau}^{t} z\left(\theta_{r-t} \omega\right) d r} d \tau d s \\
& \quad \leq \frac{C_{3}}{M}\left(t-T_{K}\right) e^{-\lambda t+C_{1} \int_{-t}^{0} z\left(\theta_{r} \omega\right) d r}\left\|\tilde{g}_{0}(\omega)\right\|_{H}^{2}+\frac{C_{2} C_{3}}{M} \int_{T_{K}}^{t} \int_{-t}^{s-t} e^{-2 z\left(\theta_{\tau} \omega\right)+\lambda \tau+C_{1} \int_{\tau}^{0} z\left(\theta_{r} \omega\right) d r} d \tau d s \\
& \quad \leq \frac{C_{3}}{M}\left(t-T_{K}\right) e^{-\lambda t+C_{1} \int_{-t}^{0} z\left(\theta_{r} \omega\right) d r}\left\|\tilde{g}_{0}(\omega)\right\|_{H}^{2}+\frac{C_{2} C_{3}}{M}\left(t-T_{K}\right) \int_{-t}^{0} e^{-2 z\left(\theta_{\tau} \omega\right)+\lambda \tau+C_{1} \int_{\tau}^{0} z\left(\theta_{r} \omega\right) d r} d \tau .
\end{aligned}
$$

By using (3.7), there exist $T_{2}(\epsilon, \omega, K(\omega))>T_{K}(\omega)$ and $M_{1}(\epsilon, \omega, K(\omega))>0$, such that if $t>$ $T_{2}(\epsilon, \omega, K(\omega))$ and $M>M_{1}(\epsilon, \omega, K(\omega))$, then

$$
\frac{C_{3}}{M} \int_{T_{k}}^{t} e^{-\lambda(t-s)+C_{1} \int_{s}^{t} z\left(\theta_{\tau-t} \omega\right) d \tau}\left\|\tilde{g}\left(s, \theta_{-t} \omega, \widetilde{g}_{0}\left(\theta_{-t} \omega\right)\right)\right\|_{H}^{2} d s \leq \frac{\epsilon^{2}}{3}
$$

By using (3.7) again, there exists $T_{3}(\epsilon, \omega, K(\omega))>0$, such that if $T>T_{3}(\epsilon, \omega, K(\omega))$, we have

$$
C_{2} \int_{T_{k}}^{t} e^{-\lambda(t-s)-2 z\left(\theta_{s} \omega\right)+C_{1} \int_{s}^{t} z\left(\theta_{\tau} \omega\right) d \tau} d s \leq \frac{\epsilon^{2}}{3}
$$

Therefore, by letting

$$
\begin{gathered}
T(\epsilon, \omega, K(\omega))=\max \left\{T_{1}(\epsilon, \omega, K(\omega)), T_{2}(\epsilon, \omega, K(\omega)), T_{3}(\epsilon, \omega, K(\omega))\right\}, \\
M(\epsilon, \omega, K(\omega))=M_{1}(\epsilon, \omega, K(\omega)),
\end{gathered}
$$

we obtain, for $t>T(\epsilon, \omega, K(\omega))$ and $M>M(\epsilon, \omega, K(\omega))$,

$$
\sum_{|i| \geq 2 M}\left|\widetilde{g}_{i}\left(t, \theta_{-t} \omega, \widetilde{g}_{0}\left(\theta_{-t} \omega\right)\right)\right|_{H}^{2} \leq \sum_{i \in \mathbb{Z}} \rho\left(\frac{|i|}{M}\right)\left|\widetilde{g}_{i}\left(t, \theta_{-t} \omega, \widetilde{g}_{0}\left(\theta_{-t} \omega\right)\right)\right|_{H}^{2} \leq \epsilon^{2}
$$

which implies

$$
\sum_{|i|>M(\epsilon, \omega, K(\omega))} \mid\left(\left.\varphi\left(t, \theta_{-t} \omega, \varphi_{0}\left(\theta_{-t} \omega\right)\right)_{i}\right|_{H} ^{2} \leq \epsilon^{2}, \quad \forall t \geq T(\epsilon, \omega, K(\omega))\right.
$$

The proof is completed.

Now, we have the main result. 
Theorem 3.6. The random dynamical system $\varphi$ generated by system (3.9) has a unique global random attractor.

Proof. It is obvious from Lemmas 3.4, and 3.5, and Proposition 2.5.

\section{Acknowledgments}

This work was supported by National Natural Science Foundation of China under Grant 11071165 and Guangxi Provincial Department of Research Project under Grant 201010LX166.

\section{References}

[1] A. Gu, "Random attractors for stochastic three-component reversible Gray-Scott system with multiplicative white noise," Journal of Applied Mathematics, vol. 2012, Article ID 810198, 15 pages, 2012.

[2] H. Mahara, N. J. Suematsu, T. Yamaguchi, K. Ohgane, Y. Nishiura, and M. Shimomura, "Three-variable reversible Gray-Scott model," Journal of Chemical Physics, vol. 121, no. 18, pp. 8968-8972, 2004.

[3] Y. You, "Dynamics of three-component reversible Gray-Scott model," Discrete and Continuous Dynamical Systems Series B, vol. 14, no. 4, pp. 1671-1688, 2010.

[4] P. Gray and S. K. Scott, "Autocatalytic reactions in the isothermal, continuous stirred tank reactor. Isolas and other forms of multistability," Chemical Engineering Science, vol. 38, no. 1, pp. 29-43, 1983.

[5] P. Gray and S. K. Scott, "Autocatalytic reactions in the isothermal, continuous stirred tank reactor. Oscillations and instabilities in the system $a+2 b \rightarrow 3 b, b \rightarrow c$," Chemical Engineering Science, vol. 39, no. 6, pp. 1087-1097, 1984.

[6] R. Kapral, "Discrete models for chemically reacting systems," Journal of Mathematical Chemistry, vol. 6, no. 2, pp. 113-163, 1991.

[7] R. Kapral and K. Showalter, Eds., Chemical Waves and Patterns, Understanding Chemical Reactivity, Springer, Berlin, Germany, 1994.

[8] S. Scott and K. Showalter, "Simple and complex reaction-diffusion fronts, ," in Chemical Waves and Patterns Understanding Chemical Reactivity, R. Kapral and K. Showalter, Eds., vol. 10, pp. 485-516, Springer, Berlin, Germany, 1995.

[9] P. W. Bates, H. Lisei, and K. Lu, "Attractors for stochastic lattice dynamical systems," Stochastics and Dynamics, vol. 6, no. 1, pp. 1-21, 2006.

[10] T. Caraballo and K. Lu, "Attractors for stochastic lattice dynamical systems with a multiplicative noise," Frontiers of Mathematics in China, vol. 3, no. 3, pp. 317-335, 2008.

[11] X. Han, W. Shen, and S. Zhou, "Random attractors for stochastic lattice dynamical systems in weighted spaces," Journal of Differential Equations, vol. 250, no. 3, pp. 1235-1266, 2011.

[12] X. Han, "Random attractors for stochastic sine-Gordon lattice systems with multiplicative white noise," Journal of Mathematical Analysis and Applications, vol. 376, no. 2, pp. 481-493, 2011.

[13] S. Zhou, "Upper-semicontinuity of attractors for random lattice systems perturbed by small white noises," Nonlinear Analysis: Theory, Methods E Applications, vol. 75, pp. 2793-2805, 2012.

[14] C. Zhao and S. Zhou, "Sufficient conditions for the existence of global random attractors for stochastic lattice dynamical systems and applications," Journal of Mathematical Analysis and Applications, vol. 354, no. 1, pp. 78-95, 2009.

[15] X. Wang, S. Li, and D. Xu, "Random attractors for second-order stochastic lattice dynamical systems," Nonlinear Analysis: Theory, Methods E Applications, vol. 72, no. 1, pp. 483-494, 2010.

[16] T. Erneux and G. Nicolis, "Propagating waves in discrete bistable reaction-diffusion systems," Physica D, vol. 67, no. 1-3, pp. 237-244, 1993.

[17] J. P. Laplante and T. Erneux, "Propagation failure in arrays of coupled bistable chemical reactors," Journal of Physical Chemistry, vol. 96, no. 12, pp. 4931-4934, 1992.

[18] L. Arnold, Random Dynamical Systems, Springer Monographs in Mathematics, Springer, Berlin, Germany, 1998. 
[19] I. Chueshov, Monotone Random Systems Theory and Applications, vol. 1779 of Lecture Notes in Mathematics, Springer, Berlin, Germany, 2002.

[20] X. Fan, "Attractors for a damped stochastic wave equation of sine-Gordon type with sublinear multiplicative noise," Stochastic Analysis and Applications, vol. 24, no. 4, pp. 767-793, 2006. 


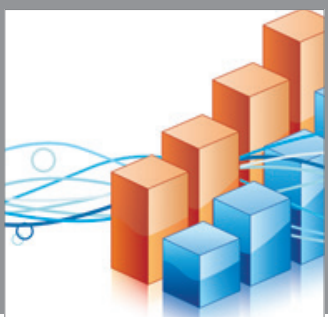

Advances in

Operations Research

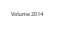

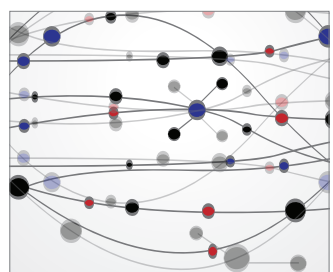

\section{The Scientific} World Journal
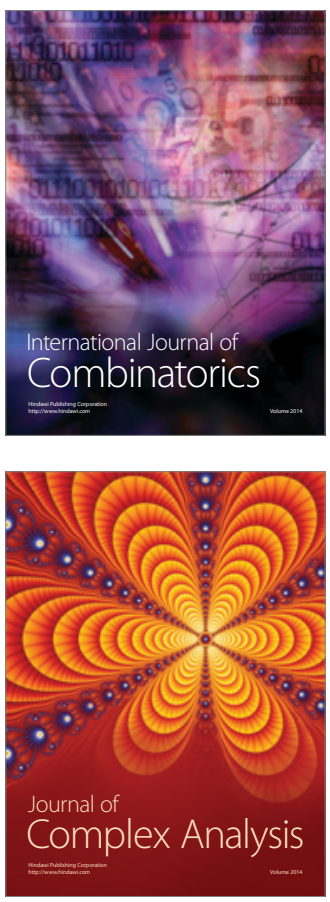

International Journal of

Mathematics and

Mathematical

Sciences
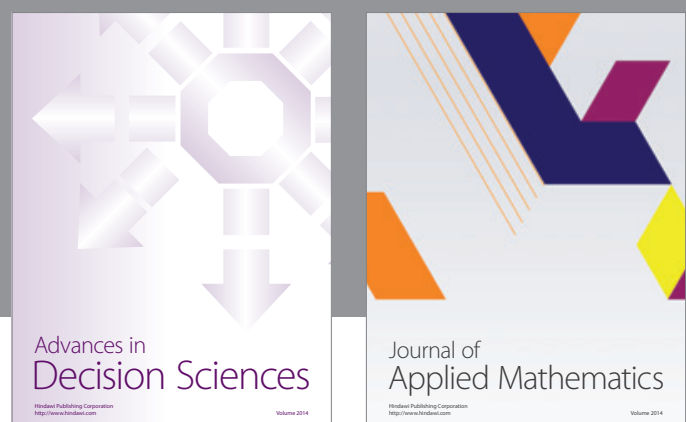

Journal of

Applied Mathematics
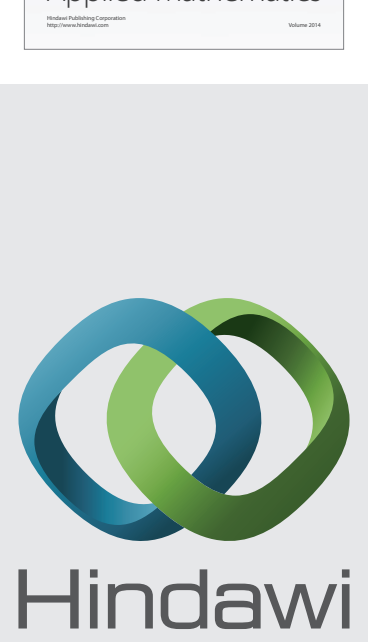

Submit your manuscripts at http://www.hindawi.com
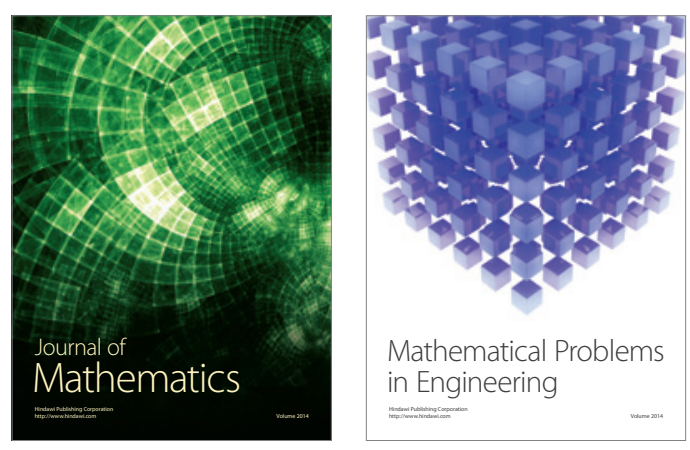

Mathematical Problems in Engineering
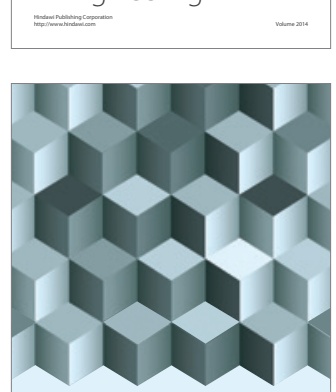

Journal of

Function Spaces
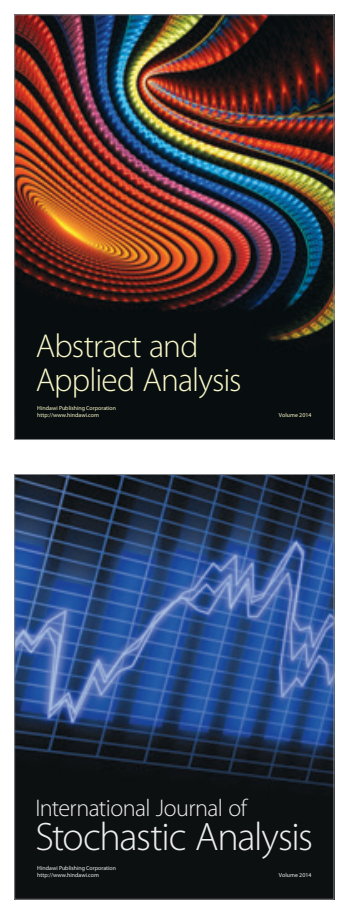

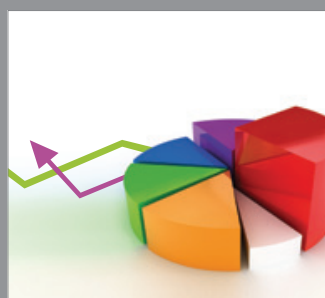

ournal of

Probability and Statistics

Promensencen
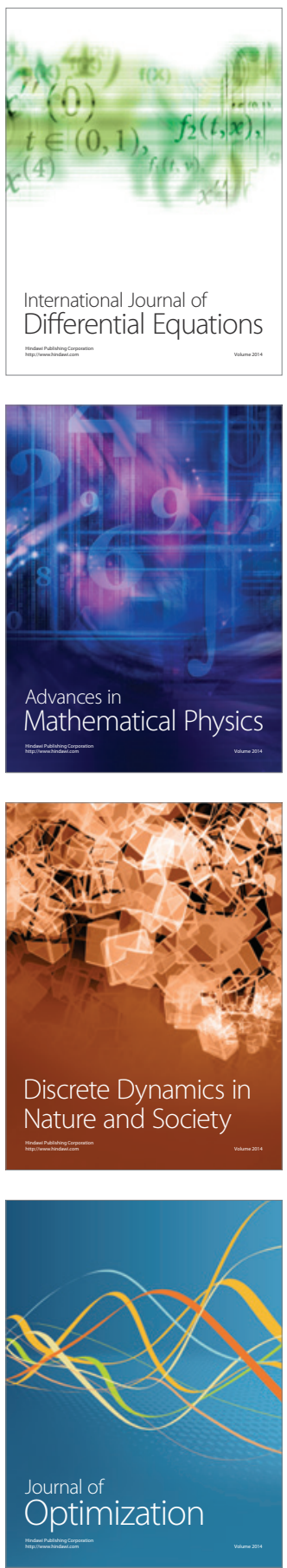